Bundesgesundheitsbl -

Tätigkeitsberichte

M. Exner ${ }^{1} \cdot$ Th. Kistemann ${ }^{1} \cdot$ G. Unger ${ }^{2} \cdot$ M. Hansis $^{3} \cdot$ A. Nassauer $^{4}$

${ }^{1}$ Hygiene-Institut der Universität Bonn . ${ }^{2}$ Umweltbundesamt, Bad Elster $\cdot{ }^{3}$ Klinik und Poliklinik, Unfallchirurgie der Universität Bonn · ${ }^{4}$ Robert Koch-Institut, Berlin

\title{
Zukünftige Pråventions- und Kontrollstrategien in der Krankenhaushygiene
}

\section{Zur Arbeit der Krankenhaushygiene- Kommission am Robert Koch-Institut}

\begin{abstract}
Und so lassen sie mich denn diesen Vortrag schließen mit dem Wunsch, daß sich die Kräfte der Nationen auf diesem Arbeitsfelde... messen mögen und daß in diesem Kampfe zum Wohle der gesamten Menschheit eine Nation die andere in ihren Erfolgen immer wieder überflügeln möge.
\end{abstract}

(Robert Koch, X. Internationaler Medizinischer Kongress, Berlin 1890) m September 1997 wurde die Kommission für Krankenhaushygiene und Infektionsprävention am Robert KochInstitut (RKI) neu konstituiert, nachdem vor Berufung der Mitglieder u.a. eine Anhörung der Arbeitsgemeinschaft der wissenschaftlichen medizinischen Fachgesellschaften (AWMF) erfolgt war. Entsprechend der Geschäftsordnung der Kommission erarbeitet sie diese Vorschläge zur Krankenhaushygiene und Infektionsprävention in Einrichtungen des Gesundheitswesens. Nach der konstituierenden Sitzung hat sich die Kommission einen Arbeitsplan gegeben, Arbeitsgruppen gebildet, die methodischen Grundlagen für die Überarbeitung und Bearbeitung von Empfehlungen festgelegt und die Überarbeitung der Richtlinie für Krankenhaushygiene und Infektionsprävention aufgenommen. Im folgenden sollen nach der Erläuterung wichtiger Begriffe die derzeitige Situation analysiert, die bisherige Arbeit der Krankenhaushygiene-Kommission rekapituliert sowie ein Ausblick auf zukünftige Entwicklungen gegeben werden.

\section{Begriffe und Inhalte}

Während der Begriff Krankenhausinfektion in der Richtlinie für Krankenhaushygiene und Infektionsprävention definiert ist [1], wird für den Begriff Krankenhaushygiene keine Definition gegeben. Unter Krankenhausinfektion (nosokomiale Infektion) wird jede durch Mikroorganismen hervorgerufene Infektion verstanden, die im kausalen Zusammenhang mit einem Krankenhausaufenthalt steht, unabhängig davon, ob Krankheitssymptome bestehen oder nicht. Eine epidemische Krankenhausinfektion (Ausbruch) liegt dann vor, wenn Infektionen mit einheitlichem Erregertyp, mit zeitlichem, örtlichem und kausalem Zusammenhang zu einem Krankenhausaufenthalt nicht nur vereinzelt auftreten. Im Entwurf für ein Infektionsschutzgesetz (Stand: März 1999) sind beide Termini im Sinne einer Legaldefinition jetzt so beschrieben:

Nosokomiale Infektion • Eine Infektion mit lokalen oder systemischen Infektionszeichen „als Reaktion auf das Vorhandensein von Erregern oder ihren Toxinen, die im zeitlichen Zusammenhang mit einem Krankenhausaufenthalt oder einer ambulanten medizinischen Maßnahme

Prof.Dr.med. M. Exner

Hygieneinstitut der Universität Bonn,

Sigmund-Freud-Str. 25, D-53105 Bonn 
steht, soweit die Infektion nicht bereits vorher bestand“( $\$ 2 \mathrm{Nr}$. 8).

„Dem Gesundheitsamt ist unverzüglich das gehäufte Auftreten nosokomialer Infektionen, bei denen ein epidemischer Zusammenhang wahrscheinlich ist oder vermutet wird, als Ausbruch nicht namentlich zu melden( $\$ 6$ Abs. 3$)$.“

Krankenhaushygiene • Hierfür wird von uns folgende Definition vorgeschlagen:

„Unter Krankenhaushygiene soll die Wissenschaft und Lehre von der Verhütung, Erkennung und Kontrolle von Gesundheitsrisiken, insbesondere von Infektionen, für Patienten und medizinisches Personal, im Krankenhaus und sonstigen medizinischen Einrichtungen verstanden werden, wobei systematische Risikoanalyse und Entwicklung von Präventions- und Kontrollstrategien wesentliche Arbeitsfelder sind.

Die Krankenhaushygiene erarbeitet Kriterien, wie Krankenhäuser und andere Einrichtungen des Gesunheitswesens geplant, gebaut, mit den Mitarbeitern in effizienter Weise organisiert, betrieben und unterhalten werden können, um sicherzustellen, daß

D keine Gesundheitsschäden, insbesondere Infektionen, auftreten (Prävention);

D aufgetretene Gesundheitsschäden und Infektionen so zeitnah wie möglich erkannt werden (Surveillance);

D diese so rasch wie möglich unter Kontrolle gebracht werden, so daß ihre Weiterverbreitung verhindert wird."

Die Ziele, Aufgaben und Strukturen der Krankenhaushygiene [2] faßt Übersicht 1 zusammen. Die Krankenhaushygiene umfaßt Maßnahmen zum Gesundheitsschutz und zur Gesundheitsförderung.

Gesundheitsschutz $\cdot$ Hierunter werden alle Maßnahmen verstanden, die durch das Verhalten des einzelnen nicht oder bedingt zu beeinflußen sind und daher von einem Gemeinwesen ergriffen bzw. sichergestellt werden müssen, um die Gesundheit der Allgemeinheit bzw. des einzelnen zu schützen. Patienten sind darauf angewiesen, daß die notwendigen hygienischen Voraussetzungen zur Infektionsprävention im Krankenhaus, in medizinischen Bereichen und Gemeinschaftseinrichtungen (Pflegeheim) ergriffen bzw. sichergestellt werden, da sie diese nicht bzw. nur marginal beeinflussen können.

Gesundheitsförderung • Unter diesen Punkt werden alle Maßnahmen verstanden, die den einzelnen durch Ausbildung und Aufklärung zu Schutz und Förderung seiner Gesundheit und $\mathrm{zu}$ einem gesundheitsgerechten Verhalten sich selbst und anderen gegenüber befähigen und motivieren. Das medizinische Personal hat hier in besonderer Weise eine hohe Verantwortung, durch entprechende Verhaltensweisen die eigene Gesundheit sowie die von Patienten und Mitarbeitern zu schützen und zu fördern. Durch Ausbildung, Schulung und Bereitstellung geeigneter baulicher und funktionaler Voraussetzungen müssen derartige gesundheitsfördernde Verhaltensweisen allerdings systematisch unterstützt werden.

\section{Verhütung nosokomialer Infektionen}

\section{Rückblick und aktuelle Konzepte}

Der Vergleich der heutigen Situation in der Krankenhaushygiene mit der vor 125 Jahren macht deutlich, welche Fortschritte und Erfolge auf diesem Gebiet erzielt wurden. Eine aufschlußreiche Situationsbeschreibung der damaligen Zeit wurde von N. von Nußbaum gegeben. Er führte aus, daß in seiner Klinik Vierfünftel aller Verwundeten oder Operierten von Nosokomialbrand befallen wurden und bei Spitalbrandkranken eine Verlängerung der Aufenthaltszeit um 70 bis 250 Tage resultierte [3]. Insbesondere nosokomiale Infektionen bestimmten somit maßgeblich die Dauer der Krankenhausbehandlung sowie die Letalität nach Operationen. Hieraus resultierte u.a. der Bedarf, ausgedehnte Krankenhausbauten zu errichten.
„Wenn Sie bedenken, daß jeder Kranke unseres Hauses täglich über einen Gulden kostet, so verzehren die Fälle von Nosokomialbrand jedes Jahr viele Tausende, denn unsere Bögen weisen bei Spitalbrand Kranken 70 bis 80,120 bis 250 Tage Aufenthaltszeit mehr nach als bei den gleichen Krankheiten ohne Nosokomialbrand.

Vierfünftel aller Verwundeten oder Operierten wurden im letzten Jahr von Nosokomialbrand befallen, so daß ich fest überzeugt bin, daß der Nosokomialbrand täglich 10 bis 20 Taler verschlingt, während der Listersche Verband vielleicht 3 Taler mehr beträgt als der gewöhnliche Verband.

Ferner kommen die Kranken bei Nosokomialbrand so sehr herab, daß man monatelang Wein, Eier, China etc. geben muß, was bei günstigem Verlauf überflüssig ist. Ich bin also fest davon überzeugt, daß in ökonomischer Beziehung Gewinn erzielt wird.

Die kleinen Stich- und Quetschwunden irgendeines Raufhandels, welche in guter Luft kaum 10 bis 20 Tage arbeitsunfähig gemacht hätten, werden durch Pyämie oder Nosokomialbrand tödlich oder machen 60 bis 70 Tage schwerkrank.

Wenn nun ein armer Dienstbote mit einer kleinen Wunde in das Spital kommt, mit einer Wunde, die bei guten Spitalverhältnissen in 14 Tagen geheilt wäre, so wird er vom Nosokomialbrand ergriffen, kommt an den Rand des Grabes, liegt unter vielen Schmerzen vielleicht 100 bis 150 Tage schwerkrank, muß chloroformiert werden, mit dem Glüheisen gebrannt werden, und wenn er endlich ganz abgemagert und noch lange arbeitsunfähig das Spital verläßt, so soll er für jeden Tag, der über 90 Tage hinausgeht, noch ein Fl. 6 kr. aus seinen Ersparnissen bezahlen oder die Hilfe seiner Gemeinde beanspruchen, während er die lange arge Krankheit doch nur dem Spitalgifte verdankt."

N. von Nußbaum [3] 


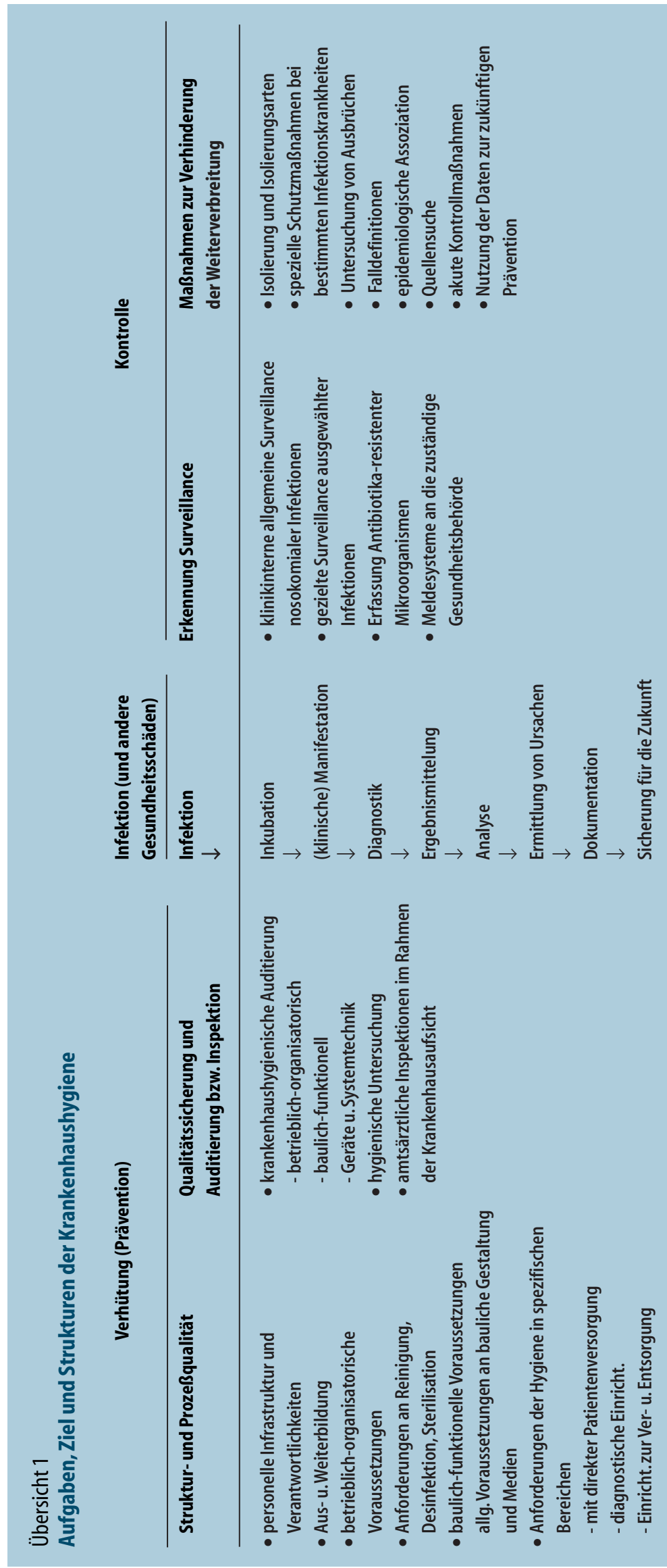

Die eindrucksvollen Erfolge durch Einführung der Händedesinfektion (Semmelweis), der Antisepsis (Lister) und der allgemeinen Sauberkeit (Nightingale) haben nicht nur die Effizienz dieser Verfahren bestätigt, sondern auch erhebliche medizinische und gesundheitsökonomische Konsequenzen gehabt. Sie waren neben der Einführung der Anästhesie die entscheidende Voraussetzung zur Etablierung der modernen Chirurgie.

Durch Verbesserung von Prävention und Kontrolle nosokomialer Infektionen wurde eine drastische Verminderung derartiger Infektionsrisiken erreicht und ein erheblicher Beitrag zur Verkürzung der durchschnittlichen Verweildauer geleistet, welche im Jahre 1994 in Deutschland 11,5 Tage betrug [4].

Nach Weinstein [5] ist jedoch in den letzten 25 Jahren in den USA die Rate nosokomialer Infektionen bemerkenswert stabil geblieben und kein weiterer Rückgang der nosokomialen Infektionsraten erreicht worden: durchschnittlich treten 5 bis 6 nosokomiale Infektionen pro 100 Neuaufnahmen auf. Da sich jedoch die durchschnittliche Krankenhausverweildauer in den letzten $20 \mathrm{Jah}-$ ren erheblich verkürzt hat, ist die Rate an nosokomialen Infektionen/1.00o Patiententage in diesem Zeitraum tatsächlich um $36 \%$ gestiegen. Während 1975 7,2 nosokomiale Infektionen/1.00o Patiententage auftraten, betrug 1995 die entsprechende Rate 9,8 (Tabelle 1). Diese Krankenhausinfektionen verursachten 1995 in den USA Kosten in Höhe von 4,5 Mrd.US-Dollar und mitbedingten mehr als 88.00o Todesfälle [5].

Hinsichtlich der Manifestationsform nosokomialer Infektionen ist ein Rückgang des Anteils von Harnwegsinfektionen und chirurgischen Wundinfektionen feststellbar, wohingegen Infektionen des unteren Respirationstraktes und nosokomiale Septikämien zugenommen haben. Es muß jedoch berücksichtigt werden, daß heute die postoperative Krankenhausverweildauer durchaus kürzer sein kann als die Inkubationszeit von Wundinfektionen (z.B. 5 bis 7 Tage bei Infektionen durch Staphylococcus aureus), so daß wahrscheinlich postoperative Wundinfektionen im Krankenhaus überproportional untererfaßt werden. 
Tabelle 1

Nosokomiale Infektionen in den USA 1975 und 1995

\begin{tabular}{llllll} 
Jahr & $\begin{array}{l}\text { Patienten- } \\
\text { aufnahmen }\end{array}$ & $\begin{array}{l}\text { Patientenverweil- } \\
\text { dauer (Tage) }\end{array}$ & $\begin{array}{l}\text { Verweildauer je } \\
\text { Patient (Tage) }\end{array}$ & $\begin{array}{l}\text { Anzahl nosokomialer } \\
\text { Infektionen }\end{array}$ & $\begin{array}{l}\text { Nosokomialinfektionen/1000 } \\
\text { Patiententage }\end{array}$ \\
\hline 1975 & 38.000 .000 & 299.000 .000 & 7,9 & 2.100 .000 & 7,2 \\
1995 & 36.000 .000 & 190.000 .000 & 5,3 & 1.900 .000 & 9,8 \\
\hline
\end{tabular}

Quelle: Weinstein, Emerg. Inf. Dis 4 (1998)

Zu den Kollektiven mit dem höchsten Risiko einer nosokomialen Infektion zählen Patienten, deren Immunkompetenz durch Alter, Grundkrankheit, Pharmakotherapie oder operative Eingriffe reduziert ist. Die demographisch bedingte Erhöhung des Durchschnittsalters sowie die zunehmende Intensität und Invasivität diagnostischer und therapeutischer Interventionen (u.a. Implantation von Fremdkörpern, Organtransplantationen und Xenotransplantationen) haben die Zahl vulnerabler $\mathrm{Pa}$ tienten im Krankenhaus erhöht. Der Anteil immunsupprimierter Patienten wird weiterhin kontinuierlich ansteigen. Darüber hinaus ist zu berücksichtigen, daß mit Zunahme ambulanter Operationen der Anteil schwer kranker Patienten in der stationären Versorgung steigt und diese aufgrund ihrer Prädisposition und Therapiebedürftigkeit auch ein höheres Risiko einer Septikämie oder einer beatmungsassoziierten Pneumonie haben. Der Trend zur Konzentration schwerstpflegebedürftiger Bewohner läßt sich im übrigen auch in Altenpflegeheimen bzw. Altersheimen feststellen.

Betrachtet man die Situation unter dem Gesichtspunkt des nosokomialen Erregerspektrums, so spielen die gramnegativen nosokomialen Infektionserreger Escherichia coli, Pseudomonas aeruginosa, Enterobacter spp. und Klebsiella pneumoniae weiterhin die führende Rolle. Bemerkenswert ist die weltweit seit den 6oer Jahren beobachtete Zunahme des Anteils antibiotikaresistenter Isolate. Besondere Bedeutung haben dabei Methicillin-resistente Staphylococcus aureus (MRSA) und Vancomycin-resistente Enterokokken (VRE). Die bedrohliche Zunahme der Resistenz bakterieller In- fektionserreger gegenüber Antibiotika gewinnt Einfluß auf die Letalität und die Mortalität nosokomialer Infektionen [6] und wird zukünftig die Krankenhaushygiene maßgeblich beschäftigen.

$S$. aureus ist einer der wichtigen Infektionserreger, sowohl von außerhalb des Krankenhauses erworbenen als auch von nosokomialen Infektionen. Durch seine Resistenz gegenüber wichtigen Antibiotika werden die Therapiemöglichkeiten entscheidend eingeschränkt. Wenn sich die gegenwärtig insbesondere in Japan und den USA beobachteten MRSA-Stämme mit zusätzlich verminderter Glykopeptid-Empfindlichkeit weiter ausbreiten, wird die Beherrschbarkeit von MRSA-Infektionen durch Wegfall der therapeutischen Glykopeptid-Option in kritischer Weise limitiert [7].

Inzidenz und Prävalenz von MRSAInfektionen können als Indikator für die Güte der Krankenhaushygiene angesehen werden, ähnlich wie das Vorkommen von E. coli im Trinkwasser einen Marker für die Güte der Trinkwasserhygiene darstellt. Von infektionsepidemiologischer Bedeutung - auch im Hinblick auf zukünftige Präventions- und Kontrollstrategien - sind die in international vergleichenden Studien festgestellten [7] erheblichen Unterschiede der MRSA-Inzidenz und -Prävalenz. Während in einigen Ländern (u.a. Japan, USA, Spanien, Italien, Frankreich, England) die MRSA-Situation kaum noch beherrschbar ist und der Anteil von MRSA an S. aureus-Isolaten aus dem Krankenhausbereich bei 20 bis $60 \%$ liegt, konnte dieser Anteil z.B. in den Niederlanden und den skandinavischen Ländern, wo strikte Präventions- und Kontrollmaßnahmen verfolgt werden, auf wenige Prozent beschränkt werden.
Wenn auch in Deutschland eine $\mathrm{Zu}$ nahme der MRSA-Inzidenz auf bis zu $8 \%$ festzustellen ist, so liegen die Raten immer noch deutlich unter denen der Hochinzidenzländer. Aus Kliniken mit effizientem Hygiene-Management werden sogar MRSA-Raten von $1 \%$ berichtet. Im intensivmedizinischen Bereich ist allerdings mit Inzidenzraten von über $10 \%$ durchaus zu rechnen [7]. Entsprechende Untersuchungen belegen aber, daß der Anteil von MRSA durch hygienische Interventionsmaßnahmen auf Intensivpflegestationen kurzfristig gesenkt werden kann ${ }^{1}[8,9]$.

Die Zunahme Antibiotika-resistenter Mikroorganismen als die Herausforderung in der Krankenhaushygiene schlechthin hat die Diskussion um wirksame Präventionsstrategien international neu entflammt. So wird insbesondere in den angelsächsischen Ländern diskutiert, ob die primär auf Kontrolle ${ }^{2}$ ausgerichteten Strategien ausreichend sind, oder ob der Akzent auf die Etablierung und Umsetzung von Präventions-

\footnotetext{
${ }^{1}$ Zu den effizienten Interventionsmaßnahmen zählen die strikte Beachtung der Händehygiene, das Tragen von Einmalhandschuhen, Schutzkitteln und Gesichtsmasken, die Desinfektion aller Materialien zur Patientenuntersuchung und -behandlung, regelmäßige Flächendesinfektion insbesondere patientennaher Bereiche sowie ggf. Raumdesinfektion nach Entlassung von MRSAPatienten [8]. Nach Einführung der 0.a. hygienischen Maßnahmen sank die Rate von MRSA auf der Intensivpflegestation einer Universitätsklinik innerhalb eines Jahres von $15 \%$ auf $4 \%$.

${ }^{2}$ Kontrolle i.S.v. Control:Maßnahmen, die auf frühzeitige Erkennung und Verhinderung der Weiterverbreitung aufgetretener Infektionen und/oder deren Infektionserregern ausgerichtet sind.
} 
maßnahmen und Hygienestandards zu verschieben ist, wozu insbesondere Schulung und Ausbildung des Personals, qualitätsgesicherte Reinigung und Desinfektion sowie hygienedienliche Gestaltung und Ausstattung von Stationen und Funktionsbereichen gezählt werden [10, 11]. Vor dem Hintergrund dieser sich abzeichnenden Neuorientierung der internationalen Strategien stellt sich aus deutscher Perspektive die Frage, inwieweit sich die 1976 etablierte „Richtlinie zur Erkennung, Verhütung und Bekämpfung von Krankenhausinfektionen" (alter Titel) in ihren Grundlinien als zukunftsorientiert bewährt hat.

Die Ziele dieser Richtlinie - Herabsetzung des Infektionsrisikos und Verhütung der Verbreitung von Krankenhausinfektionen - sind bereits in deren Vorwort formuliert. Zur Erreichung dieser Ziele wurden funktionell-bauliche Anforderungen und betrieblich-organisatorische Voraussetzungen festgelegt und die Überwachung von Arbeitsabläufen, von medizinisch-technischen Einrichtungen, von Desinfektions-, Sterilisations- und weiteren Hygienemaßnahmen eingeführt und entsprechende strukturelle Maßnahmen geregelt. Obwohl auch die systematische Erfassung von Krankenhausinfektionen in der Richtlinie gefordert ist, wurde dieser Aspekt erst in den letzten Jahren durch das Nationale Referenzzentrum für Krankenhaushygiene systematisch bearbeitet.

Die Richtlinie für Krankenhaushygiene und Infektionsprävention ist insbesondere kritisiert worden wegen

D unzureichender Transparenz bei der wissenschaftlichen Ableitung von Präventionsstrategien

D Übergewichtung von baulich-funktionellen Aspekten

D unzureichender Kriterien für die Erkennung bzw. Surveillance nosokomialer Infektionen als Voraussetzung zur Etablierung einer infektionsepidemiologischen Basis für die Ableitung von Empfehlungen

D zu langsamer Anpassung von Anlagen der Richtlinie an den aktuellen wissenschaftlichen Kenntnisstand

D fehlender Kategorisierung der Empfehlungen hinsichtlich ihrer Evidenz
D mangelhafter Strukturierung, Gliederung und Lesefreundlichkeit sowie Fehlen eines Stichwortverzeichnisses und eines Glossars.

Trotz der zum Teil berechtigten Kritik muß dennoch festgestellt werden, daß durch die Richtlinie für Krankenhaushygiene und Infektionsprävention umfassende funktionell-bauliche sowie betrieblich-organisatorische Vorgaben Empfehlungen veröffentlicht werden, die sich bei der Planung, Errichtung sowie bei der Organisation und beim Betrieb von Krankenhäusern als hilfreich erwiesen haben und zum hohen Niveau der Krankenhaushygiene in Deutschland maßgeblich beigetragen haben. Die Richtlinie hat eine sehr hohe Akzeptanz bei den Anwendern in Krankenhäusern wie auch bei den zuständigen Gesundheitsbehörden. Das hohe Niveau läßt sich u. a. an der auch im internationalen Vergleich niedrigen Rate an nosokomialen Infektionen und Inzidenz Antibiotika-resistenter Hospitalismuserreger wie MRSA ablesen [7, 12]. Darüber hinaus wurden die Berufs- bzw. Aufgabenfelder der Hygienefachkraft, des hygienebeauftragten Arztes und des Krankenhaushygienikers eingeführt und beschrieben. Diese arbeiten wesentlich präventionsorientierter als die Infection Control Nurses bzw. Infection Control Practitioners angelsächsischer Prägung [11, 13].

Für Sterilisation, Reinigung und Desinfektion wurden dezidierte Vorgaben und Wirksamkeitsprüfungen eingeführt, so daß die Voraussetzungen für Einsatz und Durchführung derartiger Maßnahmen in effizienter Weise gewährleistet sind. Im Gegensatz zu nahezu allen anderen Ländern wurden darüber hinaus Kriterien für qualitätssichernde hygienisch-mikrobiologische Untersuchungen formuliert $[1,14,15]$. Derartige Vorgaben können in einer Ära der Antibiotikaresistenzen eine herausragende Bedeutung zur Prävention therapeutisch schwer zu beherrschender nosokomialer Infektionen erlangen.

Die Effektivität krankenhaushygienischer Präventions- und Kontrollverfahren bedarf international zukünftig einer intensivierten wissenschaftlichen Evaluierung, um die bestmöglichen Strategien zu identifizieren [10, 14, 16-18]. Vergleichende Untersuchungen in deutschsprachigen und angelsächsischen Ländern sind prädestiniert zur Analyse von Charakteristika (Tabelle 2) und Unterschieden der Effizienz präventions- und surveillanceorientierter Strategien.

In Deutschland sind im Bundesseuchengesetz (BSeuchG) Vorschriften zur Verhütung übertragbarer Krankheiten im 4. Abschnitt geregelt.

In $\$ 10$ Abs. 1 heißt es:

„Werden Tatsachen festgestellt, die zum Auftreten einer übertragbaren Krankheit führen können, oder ist anzunehmen, daß solche Tatsachen vorliegen, so trifft die zuständige Behörde die notwendigen Maßnahmen zur Abwendung der dem einzelnen oder der Allgemeinheit hierdurch drohenden Gefahren."

In den Erläuterungen zu $\S 10$ des BSeuchG schreiben Schumacher und Meyn [19]:

„Ziel der Hygiene ist es, Gesunde vor Krankheit zu schützen; der Verhütung übertragbarer Krankheiten gebührt daher Vorrang vor der Bekämpfung. Während Bekämpfungsmaßnahmen das Auftreten zumindest einer Erkrankung oder doch wenigstens eines Verdachtsfalles voraussetzen und in erster Linie auf Erkennen und Verstopfen der Infektionsquelle sowie auf Unterbrechung der Infektionsketten gerichtet sind, sollen Verhütungsmaßnahmen schon im Vorfeld dafür sorgen, daß die Erreger den Menschen entweder gar nicht erreichen oder zumindest eine Infektion nicht zu einer Erkrankung führt. Es gilt also, mögliche Infektionsquellen zu erkennen und zu verstopfen, um unsere Umgebung, z.B. Nahrung - einschließlich Trinkwasser - möglichst frei von Krankheitserregern zu halten." Dieser Grundgedanke gibt der Prävention das Primat vor der Bekämpfung und wird im Folgenden als Hygiene-Konzept bezeichnet.

Im Gegensatz hierzu ist bislang das Primat in angelsächsischen Ländern traditionell auf Epidemiologie und Kontrolle ausgerichtet, worunter Interventionsmaßnahmen verstanden werden, die nach Auftreten von nosokomialen Infektionen beginnen und deren Ziel primär die Verhinderung der Weiterver- 
Tabelle 2

Aspekte der unterschiedlichen Präventionsstrategien nach dem Hygienekonzept sowie dem Infektionskontrollkonzept

Unterschiedliche Präventionsstrategien

Hygienekonzept

Infektionskontrollkonzept

Primat der Prävention

begründete Annahme von Tatsachen reicht aus, um Präventionsmaßnahme zu veranlassen

(Besorgnisgrundsatz)

theoretische Begründung

u.a. aufgrund hygienisch-mikrobiologischer

Untersuchungsergebnisse

systematische Surveillance

aktuell im Aufbau

\section{Listungswesen}

sowie systematische Reinigungs-, Desinfektions-

und Sterilisationskriterien

Infektionsrisiken aus dem unbelebten

Patientenumfeld

wird hohe Bedeutung beigemessen

funktionell-baulichen Maßnahmen

wird integrale Bedeutung zur Prävention von

Krankenhausinfektionen beigemessen

hygienische Qualitätssicherung

unabhängig vom Auftreten von Infektionen

- z.B. Hygienisch-mikrobiologische Umgebungsuntersuchungen

- interne und externe Auditierung

holistischer Ansatz

selektiver Ansatz

(C) Exner \& Kistemann 1999
Primat von Surveillance und Kontrolle

Maßnahmen werden bei Auftreten oder Häufung

von Infektionen eingeleitet

epidemiologisch basierte Evidenz

Surveillance-System

weit entwickelt

kein Listungswesen

für Desinfektionsmaßnahmen

Infektionsrisiken aus dem unbelebten

Patientenumfeld

wird geringe Bedeutung beigemessen

funktionell-bauliche Maßnahmen

werden nur marginal behandelt

hygienische Qualitätssicherung

hps. im Zusammenhang mit der Kontrolle

aufgetretener Infektionen

und für die Überprüfung ihrer Wirksamkeit entwickelt (Abb.1). Die Effizienz entsprechender Präventionsstrategien orientiert sich im Infektionskontrollkonzept an der aktuellen Reduktion von krankenhauserworbenen Infektionen, wohingegen unter dem Hygienekonzept auch die Reduktion von Risikofaktoren und potentiellen zukünftigen Infektionserregern im Umfeld des Patienten als Maßstab herangezogen werden.

Während im Infektionskontrollkonzept die Bedeutung des unbelebten patientenbezogenen bzw. -nahen Umfeldes als Infektionsreservoir für das Auftreten nosokomialer Infektionen sehr gering eingeschätzt wird und hierauf ausgerichtete Präventionsstrategien einen untergeordneten Stellenwert haben, wird im Hygienekonzept dem unbelebten Umfeld in den Präventionsstrategien ein hoher Stellenwert zuerkannt (Abb. 2) [15, 21-24]. So erklären sich auch die bereits erwähnten, in der deutschen Richtlinie für Krankenhaushygiene und Infektionsprävention festgelegten baulich-funktionellen und betrieblich-organisatorischen Anforderungen sowie Regelungen hinsichtlich Reinigung, Desinfektion und Sterilisation sowie hygienisch-mikrobiologischer Umgebungsuntersuchungen, die der Qualitätssicherung der vorgenannten Maßnahmen dienen [14].

\section{„Baulich-funktionelle Kriterien sind gleichsam die Hardware, betrieblich-organisatorische Aspekte die Software im System des Hygienekonzeptes."}

breitung ist. Vor diesem Hintergrund ist in den angelsächsischen Ländern ein sehr effizientes System zur Surveillance, der fortlaufend systematischen Sammlung, Analyse und Interpretation von Gesundheitsdaten bzw. Infektionsraten eingeführt worden. Surveillance-Systeme werden als notwendig angesehen zur Planung, Implementierung und Evaluierung von gesundheitserhaltenden bzw. fördernden Maßnahmen, wobei die zeitnahe Weitergabe gewonnener Informationen an die verantwortlichen Akteure vorausgesetzt wird $[13,20]$. Dieses Vorgehen wird im folgenden als Infektionskontroll-Konzept bezeichnet.
Hinsichtlich der Qualifikation der medizinischen Experten bestehen sinngemäße Unterschiede: Während nach dem Hygienekonzept Ärzte für Hygiene und Umweltmedizin (oder Mikrobiologen mit krankenhaushygienischer $\mathrm{Zu}$ satzqualifikation) verantwortlich sind, obliegt die Infektionskontrolle nach dem Infektionskontrollkonzept Epidemiologen, die in der Regel als fachärztlich qualifizierte Kliniker eine Zusatzausbildung in Epidemiologie absolviert haben. ${ }^{3}$

Mittlerweile wurden sehr differenzierte Anforderungen für die Entwicklung effektiver Präventionsstrategien

\footnotetext{
${ }^{3}$ Das Primat von Epidemiologie und Kontrolle in angelsächsischen Ländern wird auch deutlich in der Bezeichnung medizinischer Fachberufe, Organisationen und Journals: Infection Control Practioner, Infection Control Nurses; Hospital Infection Control Practices Advisory Committee (HICPAC); Journal of Infection Control and Hospital Epidemiology, American Journal of Infection Control oder Journal of Hospital Infection
}

${ }^{4} \mathrm{HACCP}=$ Hazard Analysis Critical Control Point 


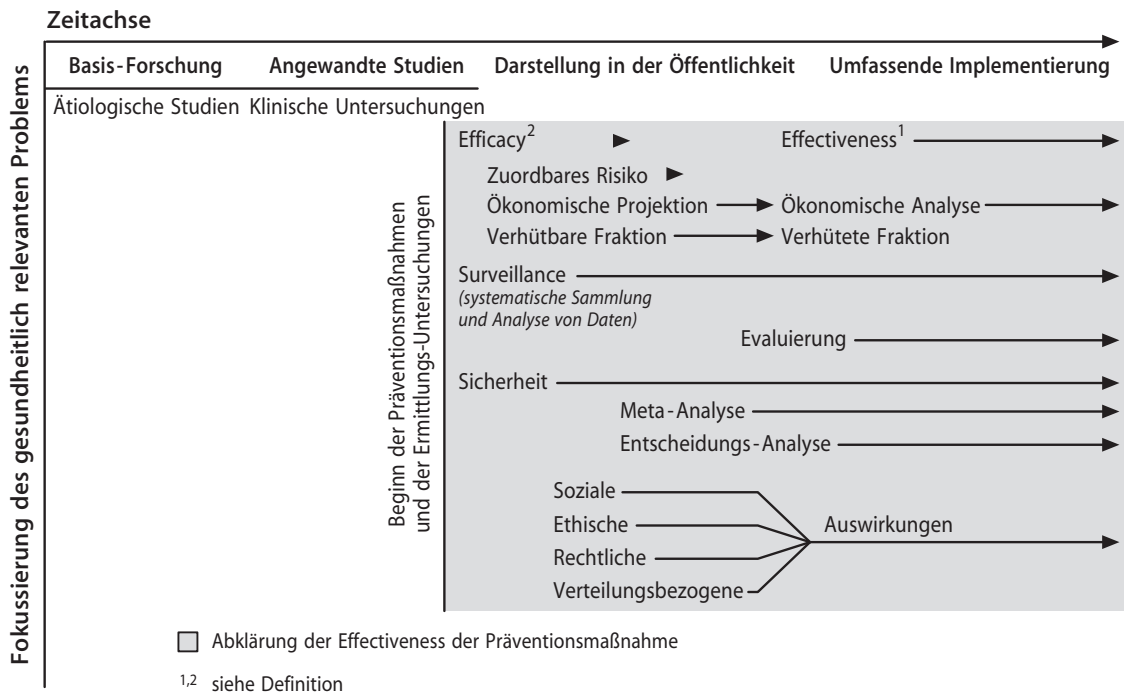

Abb. $1 \Delta$ Entwicklung von effektiven Präventionsstrategien und zeitliche Beziehung zu den Arten der Ermittlungs-Aktivitäten. Quelle: Teutsch, S.M.: A Framework for Assessing the Effectiveness of Disease and Injury Prevention. In: MMWR 41(1992), RR-3, S.5

spruch zu dem in den USA für die Raumfahrtbehörde NASA entwickelten sogenannten HACCP4-Konzept, welches mittlerweile in allen westlich-entwickelten Ländern im Lebensmittelbereich aufgrund gesetzlicher Verpflichtungen konsequent umgesetzt wird. Das HACCP-Konzept hat - in Übereinstimmung mit dem im BSeuch Geschriebenen Primat der Prävention - zum Ziel, Risikopunkte bzw. Infektionsreservoirs zu erkennen und zu eliminieren, bevor diese zu Infektionen geführt haben. Das HACCP-Konzept trägt dabei auch der betriebswirtschaftlichen Sicht Rechnung, daß in der Lebensmittelbranche, wie in allen in großen Stückzahlen für den überregionalen Markt produzierenden Industrien, qualitätsbeeinträchtigende Fertigungsmängel existentielle Konsequenzen für den Produzenten haben können. Ein Rückgang der Inzidenz von Salmonellosen und Campylobacteriosen in den USA wird mit der Einführung des HACCP-Konzeptes in Zusammenhang gesehen [25]. Somit gelten in der Lebensmittelproduktion aufgrund des HACCP-Konzeptes strengere umgebungs- und prozeßhygienische Anforderungen als dies das InfektionskontrollKonzept für das Patientenumfeld in Einrichtungen des Gesundheitswesens in den angelsächsischen Ländern vorsieht.
Ein weiterer grundlegender Unterschied betrifft die Händehygiene: Das Hygiene-Konzept favorisiert die hygienische Händedesinfektion mit Präparaten auf der Basis von Alkohol, wohingegen unter dem Infektionskontroll-Konzept in erster Linie Händewaschen mit Seife, ggf. mit Chlorhexidin bzw. PVPJod, propagiert wird [14]. Vergleichende Studien müssen zukünftig Effizienzunterschiede von Infektionskontroll- und Hygienekonzept unter epidemiologischen wie wirtschaftlichen Aspekten herausarbeiten. Hierbei ist sicherzustellen, dass herangezogene Indikatoren wie z.B. die MRSA-Rate, die beim Vergleich separat ermittelter, nationaler Raten unter dem Infektionskontroll-Konzept durchweg höher liegen (vgl. oben), unter vergleichbaren Bedingungen gewonnen werden.

Einer Harmonisierung der unterschiedlichen Strategien zur Verhütung nosokomialer Infektionen kommt wachsende Bedeutung zu, insbesondere da das zentrale Problemfeld Antibiotika-resistenter Erreger eine globale Dimension besitzt und eine vorübergehende Eindämmung im nationalen Maßstab keinen dauerhaften Erfolg darstellen wird. Innerhalb der Europäischen Union ist darüber hinaus der Aspekt der Patientensicherheit im rechtlichen Kontext der inzwischen vertraglich fixierten Bemühungen zur Angleichung der Lebensbedingungen zu berücksichtigen [14].

\section{Die Erarbeitung von Leitlinien für die Krankenhaushygiene}

Die Anforderungen an die Erarbeitung von Präventionsstrategien und Leitlinien sind in den letzten Jahren u.a. vor dem Hintergrund begrenzter finanzieller Ressourcen und juristischer Aspekte immer komplexer und differenzierter geworden [11, 13, 18, 26-28].

Nach Scriba [27] sollte das Verhältnis von Leistung und Ergebnis sowie Aufwand und Ertrag im Mittelpunkt der Bewertung stehen. Das unabdingbar Notwendige der medizinischen Versorgung muß vom lediglich wünschenswerten oder sogar überflüssigen abgegrenzt werden. Mehr Ergebnisorientierung im Gesundheitswesen setzt klare Vorstellungen über dessen Aufgaben und Ziele voraus. Nationale Gesundheitspolitik erfordert daher ein durchdachtes Zielsystem. Die Formulierung von Gesundheitszielen wird als politische Aufgabe gesehen, an der Betroffene und professioneller Sachverstand zusammenwirken müssen. Nur so lassen sich eine zielgerechte Allokation von Ressourcen erreichen, Verantwortlichkeiten festschreiben und rationalere Strategien erarbeiten. Qualität ist nach Scriba Annäherung an das medizinisch Notwendige oder Angemessene. Die große Mehrzahl der Akteure im Gesundheitswesen (Gesundheitspolitik, und -verwaltung, Interessenvertretungen, Ärzte) und der Betroffenen (Patienten) argumentiert hierbei in den Kategorien schulmedizinischer Lehre. Qualitätsleitlinien versuchen, diese Lehre auf der Basis gesicherter Erkenntnisse und/oder des Konsensus von Wissenschaft und Praxis zu überprüfen, zu objektivieren und als Ziele zu formulieren.

Auch die Entwicklung von Leitlinien auf dem Gebiet der Krankenhaushygiene bzw. Infektionskontrolle muß diesen Kriterien genügen, was jedoch mit

\footnotetext{
${ }^{5}$ Die Entwicklung von Leitlinien zur Infektionskontrolle durch die Association for Professionals in Infection Control and Epidemiology (APIC) dauert durchschnittlich zwei bis drei Jahre [28]
} 


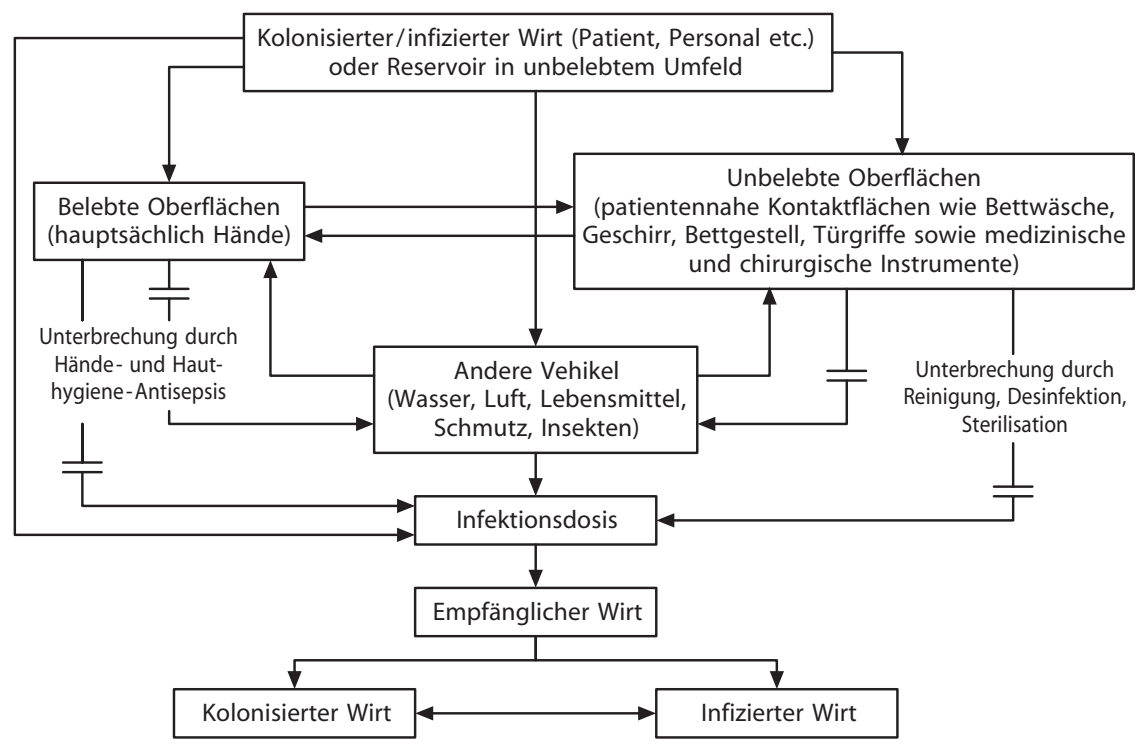

Abb. 2 ¿ Übertragung von Infektionserregern über belebte und unbelebte Oberflächen und andere Vehikel. [Quelle:Weber DJ, Reitala WA(1997) Role of Environmental Contamination in the Transmission of Vancomycin-resistent Enterococci. Inf Contr Hosp Epidem 18 S. 306-308]

einem erheblichen Aufwand an Methoden, Personal, Sachverstand und Zeit verbunden ist. ${ }^{5}$ Neben diesen systematischen Grundlagen für die Erarbeitung von Präventionsstrategien sind gesetzliche Vorgaben wie BSeuchG bzw. Entwurf des Infektionsschutzgesetzes (E-IfSG) und andere Regelungswerke wie die Unfallverhütungsvorschriften, zu berücksichtigen und die Kompatibilität der Empfehlungen mit diesen Vorgaben $\mathrm{zu}$ gewährleisten.

Anders als bei Leitlinien für Diagnostik und Therapie, die in erster Linie das Verhältnis zwischen Arzt und Patient bzw. Kostenträgern berühren, dienen Richtlinien für die Krankenhaushygiene auch den Aufsichtsbehörden (Öffentlicher Gesundheitsdienst) als Leitlinie ihrer Begehungen. Die Sicherstellung der Krankenhaushygiene ist Ländersache, weswegen die Richtlinie einer Bundesoberbehörde schon aus rechtssystematischen Gründen für medizinische Einrichtungen in den einzelnen Bundesländern nicht als rechtsverbindlich gelten kann. Die im Einzelfall notwendigen baulich-funktionellen sowie betrieblichorganisatorischen Maßnahmen und Voraussetzungen zur Prävention von Gesundheitsschäden in Krankenhäusern und anderen Einrichtungen des Gesundheitswesens müssen unter Berück- sichtigung der örtlichen Gegebenheiten und Voraussetzungen vom Krankenhaushygieniker bzw. der zuständigen Gesundheitsbehörde, unter Beachtung ggf. erlassener KrankenhaushygieneVerordnungen des jeweiligen Bundeslandes, festgelegt werden. Krankenhäusern und anderen Einrichtungen des Gesundheitswesens verbleiben im Rahmen der dargestellten Interpretation Entscheidungsspielräume. Die Richtlinie hat somit den Charakter einer Leitlinie, von der dann begründet abgewichen werden kann, wenn die konkrete Situation dies nach Würdigung der Vorgaben der Richtlinie zuläßt.

Durch die Richtlinie wird nicht von Staats wegen ein bestimmtes Verhalten ge- oder verboten. Die Richtlinie ist kein Gesetz, keine Verordnung und keine Verwaltungsvorschrift. Allerdings neigen Gerichte dazu, der Richtlinie und ihren Anlagen den Charakter von Kunstregeln (d.h. allgemein anerkannten Standards) zuzuerkennen.

Die Erfahrungen der letzten Jahrzehnte haben gezeigt, daß jederzeit mit dem Auftreten neuer Krankheitserreger gerechnet werden muß, deren epidemiologische Bedeutung, Tenazität, Desinfektionsmitteltoleranz, Übertra-
Tabelle 3

Zusammensetzung der Kommission für Krankenhaushygiene (nach Anhörung der Arbeitsgemeinschaft der Wissenschaftlichen Medizinischen Fachgesellschaften (AWMF))
Hygiene (Ärzte für Hygiene und

Umweltmedizin)

Mikrobiologie (Ärzte für Mikrobiologie und Infektionsepidemiologie) Chirurgie

Innere Medizin

Gynäkologie Anästhesie/Intensivmedizin Öffentlicher Gesundheitsdienst

Hygienefachpflege
Bärbel Christiansen, Kiel Thomas Eikmann, Gießen Martin Exner, Bonn (Vorsitz) Axel Kramer, Greifswald Georg Peters, Münster Matthias Trautman, Ulm Martin Hansis, Bonn Joachim Martius, Agatharied Ottmar Leiß, Wiesbaden Bernd Ruf, Leipzig Joachim Martius, Hausham Klaus Unertl (stellvert. Vorsitz) Heidemarie Juras, Berlin Ingeborg Kerchhoff, Hamburg Eva Maria Becker, Wiesbaden Ute Jürs, Hamburg Siegfried Niklas, Darmstadt
Teilnahme an Sitzungen mit beratender Stimme:

- Vertreterin des Bundesministeriums für Gesundheit (Ursula Niemer) - Vertreter des Bundesministeriums für Verteidigung (Heinz Bergmann) - Vertreter des Nationalen Referenzzentrums für Krankenhaushygiene (Henning Rüden) - Vertreter des Robert Koch-Instituts (Jürgen Peters, Alfred Nassauer, Götz Unger) 
gungsweise etc. zunächst unbekannt sind [29]. Die Entwicklung von Präventionsstrategien muß deshalb so ausgerichtet sein, daß auch Infektionsrisiken durch neuerkannte oder zukünftig neu auftretende Krankheitserreger für Patienten und Personal möglichst gering sind. Dieser Grundsatz wurde in der Richtlinie für Krankenhaushygiene und Infektionsprävention bei der Formulierung der Anforderungen an die Hygiene in der Zahnmedizin erstmalig berücksichtigt [30]. Hierbei wurden insbesondere die Erfahrungen zum Auftreten der Legionelleninfektion und der HIV-Infektion sowie die neuen Erkenntnisse zur Hepatitis-C-Infektion berücksichtigt $[23,30]$.

\section{Die Arbeit der Kommission für Krankenhaushygiene und Infektionsprävention am Robert Koch-Institut}

Die Kommission für Krankenhaushygiene und Infektionsprävention am Robert Koch-Institut wurde 1997 im Einvernehmen mit dem Bundesministerium für Gesundheit durch den Leiter des Robert Koch-Institutes neu berufen. Vor der Nominierung der Mitglieder wurde u.a.die Arbeitsgemeinschaft der wissenschaftlichen Fachgesellschaften (AWMF) gehört. Die Geschäftsordnung der Kommission für Krankenhaushygiene und Infektionsprävention bestimmt ausdrücklich, daß die Kommissionsmitglieder bei Ausübung ihres Ehrenamtes als Mitglied der Kommission nur ihrem Gewissen verantwortlich und zu unparteiischer Erfüllung ihrer Aufgaben sowie zur Verschwiegenheit gegenüber Dritten verpflichtet sind.

Voraussetzungen für die Mitarbeit in einer Kommission wie der Kommission für Krankenhaushygiene und Infektionsprävention sind neben der Fachkompetenz eine gemeinsame Vision (wirksame Verhütung, Erkennung und Bekämpfung von Krankenhausinfektionen), die Verpflichtung im besten Sinne zusammenzuarbeiten, Konsensusbereitschaft, Kritikfähigkeit und -bereitschaft und wechselseitiger Respekt [11].

Der Geschäftsordnung entsprechend nehmen an den Kommissionssit- zungen neben den Mitgliedern Vertreter des Bundesministeriums für Gesundheit, des Bundesministeriums für Verteidigung, des Nationalen Referenzzentrums für Krankenhaushygiene und des Robert Koch-Institutes mit beratender Stimme teil (Tabelle 3).

Die Aufgaben der Kommission für Krankenhaushygiene sind aktuell in ihrer Geschäftsordnung ( $\$ 1)$ festgelegt und im Entwurf des Gesetzes zur Verhütung und Bekämpfung von Infektionskrankheiten beim Menschen (E-IfSG, $\$ 23$ Abs. 2) ist ihre gesetzliche Verankerung vorgesehen. Dort heißt es:

„Am Robert Koch-Institut wird eine Kommission für Krankenhaushygiene und Infektionsprävention eingerichtet, die Empfehlungen zur Prävention nosokomialer Infektionen sowie zu betrieblich-organisatorischen und baulich-funktionellen Maßnahmen der Hygiene in Krankenhäusern und anderen medizinischen Einrichtungen erstellt. Die Empfehlungen der Kommission werden vom Robert Koch-Institut veröffentlicht. Die Mitglieder der Kommission werden vom Bundesministerium für Gesundheit im Benehmen mit den Obersten Landesgesundheitsbehörden berufen. Vertreter des Bundesministeriums für Gesundheit, der Obersten Landesgesundheitsbehörden und des Robert Koch-Institutes nehmen mit beratender Stimme an den Sitzungen teil.“

Voraussetzung für eine zielgerichtete Arbeit im Sinne der Geschäftsordnung und des zukünftigen Infektionsschutzgesetzes waren die Festlegung der Arbeitsgrundlagen, der Prämissen sowie des Arbeitsprogramms.

Die Kommission beschloß und empfahl dem Robert Koch-Institut, die Richtlinie für Krankenhaushygiene und Infektionsprävention weiterhin als Grundlage für die Krankenhaushygiene in Deutschland anzusehen, sie jedoch dem aktuellen Kenntnisstand anzupassen, zu straffen, auf Redundanzen zu überprüfen, und im Hinblick auf eine leichtere Handhabbarkeit um ein Stichwortverzeichnis sowie ein Glossar zu ergänzen. Die Kommission sah es als nicht gerechtfertigt an, das hohe Niveau der Krankenhaushygiene in Deutschland, zu der die bisherige Richtlinie für Kranken- haushygiene und Infektionsprävention entscheidend beigetragen hat, durch ihre Außer-Kraft-Setzung zu gefährden. Als sinnvoll wurde vielmehr angesehen, die bestehende Richtlinie zeitnah zu überarbeiten.

Vor dem Hintergrund zum Teil konkurrierender Ziele war es vorrangig, sich auf Prämissen der Krankenhaushygiene zu einigen, die zukünftig als Kriterien bei der Erarbeitung von Empfehlungen $\mathrm{zu}$ berücksichtigen sein sollen. Die Kommission formulierte hierzu folgenden Zielkatalog:

1. Schutz von Patient und Mitpatienten (Patientenschutz) vor vermeidbarer Gesundheitsgefährdung, insbesondere Infektionsrisiken;

2. Schutz des medizinischen Personals (Personalschutz) und von Beschäftigten sowie Besuchern vor vermeidbaren Gesundheitsschäden, insbesondere Infektionsrisiken im medizinischen Bereich;

3. Schutz des patientennahen Umfeldes (Flächen, Instrumente, Geräte) vor vermeidbarer Kontamination mit Infektionserregern und Sicherstellung einer validierten Asepsis, Antisepsis, Reinigung, Desinfektion und Sterilisation, insbesondere von Flächen und Instru-

\footnotetext{
${ }^{6}$ Evidenz: Deutsche Übernahme des englischen Begriffes „evidence"; Evidentsein, die unmittelbare und vollständige Einsichtigkeit, Deutlichkeit und Gewißheit (Duden Fremdwörterbuch 1998). Evidenz in der englisch-sprachigen medizinischen Literatur hingegen bezeichnet „externe, klinisch relevante Forschungsergebnisse, die als wissenschaftlich-empirische Grundlage ärztlichen Handelns dienen." Diese kommt zum einen aus der medizinischen Grundlagenforschung, zum anderen aus der patientenorientierten klinischen Forschung (z.B. bezüglich der Validität und Präzision diagnostischer Tests, der Power prognostischer Marker; der Wirksamkeit und Sicherheit therapeutischer, rehabilitativer und präventiver Maßnahmen) [26].

${ }^{7}$ Effizienz:Verbesserung eines gesundheitsbezogenen Ergebnisses, welche durch eine Präventionsstrategie durch Fachleute (Spezialisten, Experten) unter idealen Umständen (Bedingungen) erreicht werden kann.

${ }^{8}$ Effektivität:Verbesserung eines gesundheitsbezogenen Ergebnisses, welches durch eine Präventionsstrategie unter normalen Umständen mit normal ausgebildeten Mitarbeitern erzielt werden kann.
} 
menten, die direkt oder indirekt Kontakt mit Patient oder Personal haben; 4. Ableitung von Evidenz-basierten Empfehlungen ${ }^{6}$, wo dies möglich ist; 5. Berücksichtigung von Effizienz ${ }^{7}$, Effektivität ${ }^{8}$ und Praktikabilität der Empfehlungen und deren Akzeptanz beim medizinischen Personal;

6. Berücksichtigung ökonomischer Aspekte (Aufwand an Personal, Material und baulich-funktionellen Bedingungen);

7. Berücksichtigung ökologischer Aspekte und des Ressourcenschutzes;

8. Gewährleistung der Vereinbarkeit mit gesetzlichen Bestimmungen, Verordnungen sowie Empfehlungen in Deutschland und der Europäischen Union, die den krankenhaushygienischen Bereich berühren.

Dem Patientenschutz wird die höchste Priorität beigemessen, da der Patient nur sehr eingeschränkte Möglichkeiten hat, sich aktiv vor exogener Infektionsgefährdung zu schützen und sich darauf verlassen muß, daß die hierzu notwendigen Maßnahmen im Sinne des Gesundheitsschutzes sichergestellt sind. Dem Schutz des medizinischen Personals sowie von übrigen Beschäftigten (Personalschutz) kommt ebenfalls sehr hohe Priorität zu. Da das Personal sich durch sein Verhalten maßgeblich vor berufsbedingten Gesundheitsgefährdungen und Infektionen schützen kann, sind Ausbildung, Training und Motivation besonders bedeutsam.

Der Schutz des patientennahen Umfeldes vor vermeidbarer Kontamination und die Gewährleistung einer validierten Asepsis, Antisepsis, Reinigung, Desinfektion und Sterilisation haben nach dem in Deutschland verfolgten Hygiene-Konzept, wie oben ausgeführt, einen sehr hohen Stellenwert; dies wird von der Kommission in seiner Bedeutung unverändert anerkannt. Die baulich-funktionellen und betrieblich-organisatorischen Voraussetzungen hierzu müssen zur Gewährleistung der Qualitätssicherung regelmäßig überprüft werden.

Die Evidenz von Maßnahmen soll, sofern valide epidemiologische Untersuchungen vorliegen, berücksichtigt werden. $\mathrm{Zu}$ weiten Teilbereichen der Krankenhaushygiene, insbesondere zu bau-

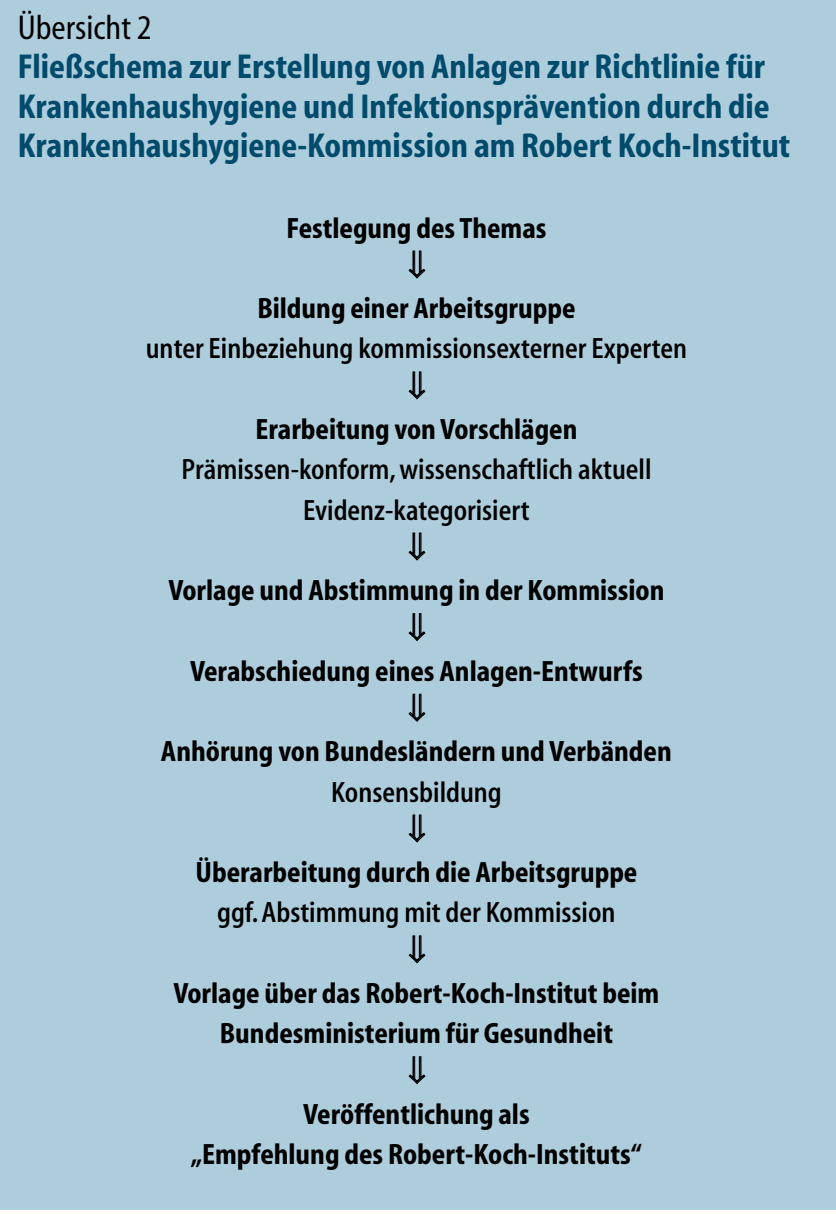

lich-funktionellen und betrieblich-organisatorischen Maßnahmen fehlen jedoch häufig entsprechende evidenz-basierte epidemiologische Studien, so daß auch die theoretische Begründung, die sich an den o.a. Zielen und Prämissen orientiert, Grundlage für Empfehlungen sein kann. Effizienz und Effektivität werden heute vor dem Hintergrund knapper Kassen in einem wesentlich stärkeren Maße hinterfragt, als dies in früheren Jahren der Fall war. Die Kommission sieht die Notwendigkeit, ökonomische Kriterien nach Möglichkeit mit zu berücksichtigen, ist jedoch der Auffassung, daß bei Fehlen entsprechender Daten notwendige hygienische Vorgaben und Empfehlungen nicht zurückgestellt werden dürfen, bis entsprechende evidenzbasierte Untersuchungen vorliegen. Alle empfohlenen Maßnahmen müssen hinsichtlich ihrer Praktikabilität überprüft sein, da hiervon auch die Akzep- $\tan z$ beim medizinischen Personal in der Umsetzung entscheidend abhängt.

Ökologische Ziele sind zu berücksichtigen, stehen jedoch in der Wertigkeit hinter den Anforderungen zur Sicherstellung des Patientenschutzes und des Personalschutzes zurück. Gesundheitsschutz hat im medizinischen Bereich Vorrang vor Umweltschutz. Um widersprüchliche Regelungen zu vermeiden, müssen die entsprechenden Empfehlungen im Hinblick auf die Vereinbarkeit mit gesetzlichen Bestimmungen, wie z.B. dem BSeuchG oder E-IfsG, oder anderen verbindlichen Regelungen wie den Unfallverhütungsvorschriften abgestimmt werden.

Das Schutzziel der Prävention ist im medizinischen Bereich noch zu wenig verankert, und bisweilen besteht beim Personal immer noch die Auffassung, Infektionen im Krankenhaus seien schicksalhaft und müssen hingenom- 
men werden. Hierdurch ist die Bereitschaft zur konsequenten Ausschöpfung verfügbarer Maßnahmen zur Prävention von krankenhauserworbenen Infektionen und anderen Gesundheitsschäden beeinträchtigt. Andererseits muß aber auch vermieden werden, daß die Umsetzung krankenhaushygienischer Empfehlungen zu übertriebenen, nicht problemgerechten Maßnahmen führen können. Neue Techniken zur Festlegung von Risikobereichen wie das erwähnte HACCP-Konzept müssen verstärkt in die Krankenhaushygiene eingeführt werden. Baulich-funktionelle sowie betrieblich-organisatorische Maßnahmen müssen sich ergänzen.

Bei der Erarbeitung von Empfehlungen für die Richtlinie für Krankenhaushygiene und Infektionsprävention verwendete man bislang zur Verdeutlichung der Verbindlichkeit oder Notwendigkeit von einzelnen Empfehlungen modale Hilfsverben wie soll, sollte oder muß. Um einerseits dem Anwender in Klinik und Praxis vor Ort Entscheidungsspielräume zu verdeutlichen und andererseits Gesundheitsbehörden ein Evidenzkriterium einzelner Empfehlungen an die Hand zu geben, ist es sinnvoll und inzwischen international üblich und bewährt, auf der Basis von wissenschaftlicher Evidenz, theoretischer Begründung, Anwendbarkeit und ökonomischen Aspekten eine Kategorisierung der Empfehlungen vorzunehmen [31]. Deshalb hat die Kommission vorgeschlagen, zukünftige Empfehlungen in Anlehnung an die von den Centers for Disease Control and Prevention (CDC, Atlanta) verwendeten Kriterien zu kategorisieren. Abweichend wurde allerdings die Einführung einer vierten Kategorie empfohlen, um die Kompatibilität mit gesetzlichen Bestimmungen, Verwaltungsvorschriften und anderen rechtsverbindlichen Verordnungen $\mathrm{zu}$ gewährleisten und auf diese hinzuweisen, wenn sich hierin spezifische Regeln finden, die krankenhaushygienische Empfehlungen berühren.

Die Definitionen der einzelnen Kategorien lauten:

D Kategorie I A - nachdrückliche Empfehlung für alle Krankenhäuser: Diese Empfehlungen basieren auf gut kon- zipierten experimentellen oder epidemiologischen Studien.

D Kategorie I B - nachdrückliche Empfehlung für alle Krankenhäuser: Diese Empfehlungen werden von Experten und aufgrund eines Konsensbeschlusses der Kommission für Krankenhaushygiene und Infektionsprävention am Robert Koch-Institut als effektiv angesehen und basieren auf gut begründeten Hinweisen für deren Wirksamkeit. Eine Einteilung in die Kategorie I B kann auch dann erfolgen, wenn wissenschaftliche Studien möglicherwei- se hierzu nicht durchgeführt wurden.

D Kategorie II - Einführung/Umsetzung in vielen Kliniken empfohlen: Diese Empfehlungen basieren teils auf hinweisenden klinischen oder epidemiologischen Studien, teils auf nachvollziehbaren theoretischen Begründungen, die in einigen, aber nicht allen Kliniken anzuwenden sind.

D Kategorie III - keine Empfehlung oder ungelöste Fragen: Maßnahmen, über deren Wirksamkeit nur unzureichende Hinweise vorliegen oder bislang kein Konsens besteht.

\section{Tabelle 4}

Arbeitsgruppen der Krankenhaushygiene-Kommission am Robert Koch-Institut, deren Leiter und Themengebiete

Arbeitsgruppe $\quad$ Themengebiete
Vorsitz

\section{Intensivmedizin}

Klaus Unertl,Tübingen

\section{Operative Medizin}

Martin Hansis, Bonn

Intravasale Systeme

Matthias Trautmann, Ulm

Harnwegskatheterismus

Joachim Martius, Agatharied

Surveillance nosokomialer Infektionen Alfred Nassauer, Berlin

Ausbrüche nosokomialer Infektionen

Andrea Ammon, Berlin

Händehygiene

Axel Kramer, Greifswald

Nosokomiale Infektionserreger

Georg Peters, Münster

Gliederung und Gestaltung der zukünftigen Richtlinie für Krankenhaushygiene und Infektionsprävention Martin Exner, Bonn

Alfred Nassauer, Berlin
Organisation und Betrieb von Intensiveinheiten Überprüfung baulich-funktioneller Vorgaben für Intensiveinheiten

Prävention der nosokomialen Pneumonie

baulich-funktionelle Anforderungen an OP-Abteilungen Anforderungen an Betrieb und Organisation von OPEinheiten

Anforderungen an Wundverbände und Verbandswechsel

Prävention und Kontrolle Katheter-assoziierter intravasaler Infektionen

Prävention und Kontrolle Katheter-assoziierter Harnwegsinfektionen

Surveillance nosokomialer Infektionen und Erfassung von Krankheitserregern mit speziellen Resistenzen und Multiresistenzen

Leitlinie zur Untersuchung von Ausbrüchen nosokomialer Infektionen

Erstellung von Anforderungen an die Händehygiene

Mitteilung zur Prävention und Kontrolle Methicillin-resistenter Staphylococcus aureus-Stämme (MRSA) in Krankenhäusern und anderen medizinischen Einrichtungen

Gliederung und Strukturierung der zukünftigen Richtlinie für Krankenhaushygiene und Infektionsprävention, Stichverzeichnis, Glossar 
D Kategorie IV: Anforderungen, Maßnahmen und Verfahrensweisen in Krankenhäusern, die aufgrund gesetzlicher Bestimmungen, autonomen Rechts oder Verwaltungsvorschriften vorgeschrieben sind.

Aus der Definition der Kategorien geht hervor, dass Empfehlungen auch bei Fehlen wissenschaftlicher Studien herausgegeben werden können, wenn sie von Experten empfohlen werden und ein Konsensusbeschluß der Krankenhaushygiene-Kommission vorliegt (Kategorie I B).

Kategorie III bedeutet nicht, daß entsprechende Maßnahmen nicht durchgeführt werden sollten. Es besteht jedoch hierzu bislang kein Konsens, weswegen man bei der Erstellung von Handlungsanweisungen in Kliniken und Praxen in diesem Bereich vor Ort entscheiden muß, ob eine Maßnahme sinnvoll ist oder nicht.

Die Erstellung von Anlagen zur Richtlinie für Krankenhaushygiene und Infektionsprävention erfolgt in mehreren Arbeitsschritten (Übersicht 2). Die Kommission legt die zu bearbeitenden Themengebiete fest und bildet entsprechende Arbeitsgruppen. Zur Erzielung der bestmöglichen, auf dem neuesten wissenschaftlichen Kenntnisstand basierenden Empfehlungen können auf Vorschlag von Kommissionsmitgliedern und Bestätigung durch die Kommission Experten auch von außerhalb als Mitglieder in Arbeitsgruppen berufen werden. Bei der Erarbeitung von Vorschlägen ist zu unterscheiden zwischen solchen zu bereits bestehenden Anlagen zur Richtlinie für Krankenhaushygiene und Infektionsprävention und solchen zu neuen, ggf. aus aktuellem Anlaß herausgegebenen Empfehlungen, die bislang nicht in Anlagen zur Richtlinie gegeben werden.

Um zu gewährleisten, daß die Empfehlungen der Richtlinie fortlaufend dem aktuellen Stand der wissenschaftlichen Erkenntnis und Entwicklung entsprechen, müssen ihre Anlagen in regelmäßigen Abständen - idealerweise in einem Rhythmus von drei bis fünf Jahren [26] bzw. je nach Aktualität unverzüglich - überpüft, angepaßt und ggf. vollständig neu erarbeitet oder zurückgezogen werden. Empfehlungen vorhandener Anlagen zur Richtlinie für Krankenhaushygiene und Infektionsprävention können zur Erhöhung der Arbeitseffizienz hinsichtlich einzelner Teilaspekte überprüft werden, die einer Änderung oder Anpassung bedürfen, ohne daß eine vollständige Überarbeitung der gesamten Anlage notwendig wird. Sie werden dann unter Berücksichtigung der Fachliteratur, analoger Leitlinien und Empfehlungen anderer Länder und unter Zugrundelegung der o.g. Prämissen und Kategorien überprüft. Sofern erforderlich, werden eine weitergehende Literaturrecherche, Qualitätsbewertung der recherchierten Literatur, sowie eine Literaturanalyse durchgeführt. Hiernach werden die Schlüsselaussagen festgelegt und nach Verknüpfung von Empfehlung und Evidenz die Kategorien festgelegt.

Die Arbeitsgruppe erstellt auf dieser Grundlage einen Entwurf, der der Kommission zur Abstimmung vorgelegt wird. Nach seiner Verabschiedung wird ein schriftliches oder mündliches Anhörungsverfahren von Bundesländern und Verbänden unter Moderation des Arbeitsgruppenleiters durchgeführt. Danach werden die Empfehlungen ggf. durch die jeweilige Arbeitsgruppe gemeinsam mit dem Robert Koch-Institut überarbeitet und dem Bundesministerium für Gesundheit vorgelegt. Nach $\mathrm{Zu}$ stimmung durch das Bundesministerium für Gesundheit werden sie als „Empfehlungen des Robert Koch-Institutes“ veröffentlicht.

Sofern zu einem Problemfeld nicht eine bestehende Anlage ergänzt und überarbeitet, sondern eine vollständig neue zu verfassen ist, müssen ggf. der Umfang der Literaturrecherchen und die Risikoanalyse ausgedehnt werden. Prinzipiell ist jedoch der Ablauf - wie im Fließschema dargestellt - gleich. Bei der Erstellung neuer Empfehlungen und Anlagen wird entweder auf systematische Übersichtsarbeiten zurückgegriffen oder diese ggf. erstellt oder in Auftrag gegeben. Ziel dieses Verfahrens ist die Reduzierung systematischer Fehler bei der Suche, Identifizierung, Auswahl, Bewertung, Zusammenfassung und Inter- pretation aller relevanten Studien zu einem bestimmten Thema.

Neben der Festlegung von Prämissen und Zielen der Kommission für Krankenhaushygiene und Infektionsprävention wurde ein Prioritätenkatalog vorrangig zu bearbeitender Themenbereiche bzw. vorrangig zu überarbeitender Anlagen erstellt. Zur Bearbeitung der entsprechenden Themengebiete wurde die Bildung von Arbeitsgruppen beschlossen. Diese stehen unter Leitung eines Kommissionsmitglieds, der weitere, für die jeweilige konkrete Fragestellung in besonderer Weise ausgewiesene Experten in die Arbeitsgruppe beruft. Seit 1997 wurden bislang neun verschiedene Arbeitsgruppen berufen (Tabelle 4).

Die neu berufene Kommission hat seit 1998 folgende Empfehlungen veröffentlicht:

D Kommentar zur Anlage zu Ziffer 4.3.4 „Anforderungen der Hygiene an die funktionelle und bauliche Gestaltung von Einheiten für Intensivmedizin“; Abmessung für Krankenräume;

D Anforderungen der Hygiene in der Zahnmedizin

D Infektionsprävention beim Transport von Patienten mit offener Lungentuberkulose

D Empfehlungen zum Vorgehen bei der Verlegung von Patienten, die mit Methicillin-resistenten Staphylococcus aureus (MRSA) infiziert oder besiedelt sind

D Empfehlungen zur Prävention und Kontrolle Katheter-assoziierter Harnwegsinfektionen.

Fertiggestellt und verabschiedet bzw.im Anhörungsverfahren befinden sich folgende Empfehlungen:

D Liste der Erreger mit speziellen Resistenzen und Multiresistenzen (gemäß E-IfSG $\$ 23$ Abs. 1)

D Mitteilung zur Prävention und Kontrolle von Methicillin-resistenten Staphylococcus aureus-Stämmen in Krankenhäusern und anderen Einrichtungen des Gesundheitswesens

- Anforderungen der Hygiene an die funktionell-bauliche Gestaltung von Operationsabteilungen, von Einheiten für kleine operative Eingriffe sowie Untersuchungs- und Behandlungsräumen für operative Fachgebiete 
D Anforderungen an die Händehygiene;

D Prävention der nosokomialen Pneumonie.

In Bearbeitung bzw. in Vorbereitung befinden sich derzeit Empfehlungen zur

- Infusionstherapie und Katheterisierung von Gefäßen

D Neubearbeitung der Richtlinie für Krankenhaushygiene und Infektionsprävention (Basisrichtlinien)

D Surveillance nosokomialer Infektionen

D Untersuchung von Ausbrüchen nosokomialer Infektionen

- Anforderung an die Aufbereitung von Medizinprodukten.

Bei allen Arbeiten hat das Nationale Referenzzentrum für Krankenhaushygiene durch seine Mitwirkung und Anhörung maßgeblich die Arbeit der Kommission unterstützt.

\section{Schlußbemerkung}

Prävention, Surveillance und Kontrolle müssen stets dem neuesten wissenschaftlichen Kenntnisstand angepaßt sein, das methodische Instrumentarium muß fortlaufend verfeinert werden. Die wissenschaftliche Untersuchung und Evaluierung darf jedoch niemals Selbstzweck sein, sondern muß den Prämissen der Krankenhaushygiene - Schutz von Patient und Personal vor vermeidbaren Gesundheitsgefahren - dienen. Erfolge bei der Verhütung von Gesundheitsschäden und der Vermeidung der Weiterverbreitung von Infektionen, insbesondere durch Antibiotika-resistente Mikroorganismen, sind das entscheidende Maß im internationalen Wettbewerb um die besten Strategien für einen wirksamen Gesundheitsschutz und eine wirksame Gesundheitsförderung.

\section{Literatur}

1. Robert Koch-Institut (1976) Richtlinie für Krankenhaushygiene und Infektionsprävention. Gustav Fischer, Stuttgart

2. NN (1989) Grundsätze und Maßnahmen zur Optimierung der Hygiene in den Krankenhäusern - DKG - Vorstandsbeschluß. Das Krankenhaus 91:607-608

3. von Nussbaum M (1875) Listers große Erfindung. Ärztl Intelligenzblatt 5:41-44

4. Statistisches Bundesamt (1996) Statistisches Jahrbuch 1996 für die Bundesrepublik Deutschland. Metzler-Poeschel, Stuttgart

5. Weinstein RA (1998) Nosocomial infection update. Emerg Inf Dis 44:416-420

6. Rubin RJ, Harrington CA, Poon A, Dietrich K, Greene JA, Moiduddin A (1999) The economic impact of staphylococcus aureus infection in New York city hospitals. Emerg Inf Dis 5: 9-17

7. NN Mitteilung zur Prävention und Kontrolle von Methicillin-resistenten Staphylococcus aureus-Stämmen (MRSA) in Krankenhäusern und anderen medizinischen Einrichtungen. Mitteilung der Kommission für Krankenhaushygiene und Infektionsprävention am RKI - im Druck befindlich

8. Schmitz J, Verhoef J, Idel H, Hadding K, Heinz HP, Jones ME (1999) Impact of hygienic measures on the development of methicillinresistance among staphylococci between 1991 and 1996 in an university hospital. J Hosp Infect 41:237-239

9. Schweitzer S, Billig J (1999) MRSA-Infektion auf einer Intensivabteilung. Epid Bull 6/99, 195-196

10. Barrett SP, Mummery RV, Chattopadhyaj B (1998) Trying to control MRSA causes more problems than it solves. J Hosp Inf 39:85-93

11. Lovett LL, Massanari RM (1999) Role of surveillance in emerging health systems: measurement is essential but not sufficient. Am J Inf Control 27: 135-140

12. Gastmeier P, Sohr G, Geffers C, Koch J, Rüden H, Nassauer A, Daschner F (1998) Identifikation von krankenhaushygienischen Problemen durch Infektionsstatistiken. Erste Ergebnisse eines Surveillance-Systems für Intensivstationen. InfFo: 1/98, 19-21

13. Scheckler WE, Brunhall D, Buck AS et al. (1998) Requirements for infrastructure and esential activities of infection control and epidemiology in hospitals: A consensus Panel report. Am J Infect Control 26:47-60

14. Exner M, Harke H-P, Gundermann KO (1999) Präventionsstrategien in der Krankenhaushygiene - Die Notwendigkeit einer Harmonisierung in Europa oder:Welcher ist der beste Weg nach Rom? Hyg Med 24: $12-20$

15. NN (1994) Kontrolle der Biokontamination. Teil 1: Grundlagen und Bestimmungen kritischer Kontrollpunkte in Risikozonen. pr EN: 1632-1
16. Martone WJ (1998) Spread of Vancomycinresistant Enterococci: Why did it happen in the United States. Infect Control Hosp Epidemiol 19:539-545

17. Goossens H (1998) Spread of Vancomycinresistant Enterococci. Differences between the United States and Europe. Infect Control Hosp Epidemiol 19:546-551

18. NN (1992) A framework for assessing the effectiveness of disease and injury prevention. MMWR 41: RR-3

19. Schumacher W, Meyn E (1992) Bundesseuchengesetz; Neue kommunale Schriften 43-4, überarbeitete Auflage. Deutscher Gemeindeverlag

20. Gaynes RP, Horan TC (1996) Surveillance of nosocomial infections. In: Mayhall CG (ed) Hospital epidemiology and infection control. Williams \& Wilkins, Baltimore, pp 1017-1031

21. Weber DJ, Rutala WA (1997) Role of environmental contamination in the transmission of Vancomycin-resistant Enterococci. Inf Control Hosp Epidemiol 18:306-309

22. NN (1995) Recommendations for preventing the spread of Vancomycin-resistance - recommendations of the hospital infection control practices advisory committee (HICPAC). MMWR 44: RR-12

23. Yu VL (1998) Resolving the controversy on environmental cultures for Legionella: $A$ modest proposal. Inf Control Hosp Epidemiol 19:893-897

24. Rüden H, Daschner F (1998) Umgebungsuntersuchungen im Krankenhaus. Stellungnahme des NRZ für Krankenhaushygiene auf Anfragen. InfFo III + IV 98:83-84

25. NN (1999) Incidence of foodborne illnesses: preliminary data from the foodborne diseases active surveillance network (food net) United States 1998. MMWR 48: 189-195

26. Underwood MA, Pirwitz S (1999) APIC guidelines committee: Using science to guide practice. Am J Inf Control 27:141-144

27. Scriba PC (1999) Immer dringender: Evaluation von Gesundheitszielen und Leitlinien. Dtsch Ärztebl 96: C-636-638, A-910-914

28. Xelou A, Kostoric-Cilic L, Ollenschläger G (1999) Nutzermanual zur Checkliste:,Methodische Qualität von Leitlinien". Zentralstelle der deutschen Ärzteschaft zur Qualitätssicherung in der Medizin. 1.Version

29. Exner M, Kistemann T Bedrohung durch Infektionskrankheiten? Risikoeinschätzung und Kontrollstrategien aus Sicht des Öffentlichen Gesundheitswesens. Dtsch Ärzteblatt (im Druck)

30. Kommission für Krankenhaushygiene und Infektionsprävention am Robert Koch-Institut (1998) Anforderungen an die Hygiene in der Zahnmedizin. Bundesgesundheitsbl 41: 363-369

31. Tablan OC et al. (1994) Guideline for prevention of nosocomial pneumonia. Inf Control Hosp Epidem 15:587-62 